|⿴囗口回回回

PHILOLOGIA CLASSICA
回回回回回

VOL. 11 - FASC. 1. 2016

UDC $821.124+82-1$

\title{
VON ANDERS SUNESEN ZU HENRY DETHICK: KONTINUITÄTEN DES MITTELLATEINISCHEN IN DER FRÜHEN NEUZEIT
}

\author{
Bernd Roling \\ Freie Universität Berlin, Institut für Griechische und Lateinische Philologie, \\ Habelschwerdter Allee 45, 14195, Berlin, Deutschland; berndroling@zedat.fu-berlin.de
}

At the beginning of the 18th century the Helmstedt scholar Polycarp Leyser published his 'History of Medieval Latin Poetry', to which he added a treatise to defend the medieval Latin against the charge of the so called 'barbarism'. The young professor's 'History' received more than evil reviews. The medieval Latin poetry, as Leyser's opponents claimed, was burdened with neologisms, typology, and scholastic speculation. Nevertheless, the paper offers two examples, taken from the highly controversial genre of biblical poetry, to demonstrate that although contemned by the majority of humanists, medieval Latin could be accepted by 17th and 18th century poets without further obstacles. First is a poem on the creation, the 'Hexaemeron' written by Anders Sunesen, archbishop of Lund in Denmark (1201-1224); although condemned as 'barbaric', the poem was not only praised by contemporary scholars as masterpiece, but could even serve as an authority for Swedish biblical poetry of the 18th century. Even more striking is the example of 'Feriae sacrae' by the English-Latin poet Henry Dethick (1577), a highly speculative piece of biblical poetry, which was in fact complete plagiarism of the 'Hypognosticon', a poem written by Laurence of Durham in the 12th century. Comparing the original version with its early modern 'adaptation', it can be demonstrated, that the main part of the text could be accepted even in the 17th century as 'modern' and ambitious. Only a few pieces of the work Henry had to exclude, because there were not coherent any longer to the dogmatics of the Elizabethan age.

Keywords: Medieval Latin poetry, Henry Dethick, Andreas Sunesen, Latin literature in the $18^{\text {th }}$ century.

\section{Einleitung: Mittellatein als Skandalon des 18. Jahrhunderts}

Mittellateiner zu sein, konnte im 18. Jahrhundert ein undankbares Geschäft sein. Im Jahre 1721 schreibt der Helmstedter Professor Polycarp Leyser IV. seine ,Geschichte der lateinischen Poesie des Mittelalters', ein Pionierwerk, dessen ganzer Wert erst im 19. Jahrhundert wirklich erkannt wurde ${ }^{1}$. Eine lange Geschichte der konfessionell geprägten Polemik hatte die Auseinandersetzung mit der mittellateinischen Literatur zuvor begleitet: viele Reden und protestantische Invektiven hatten, wie Leyser wußte, die mittelalterliche Latinität zuvor als barbarisch und von Grammatikfehlern und Neologismen strotzend

${ }^{1}$ Leyser 1721. Eine unveränderte Neuausgabe erschien in Halle 1741. Der Erstdruck erschien als Reprint (2 Bde.), Bologna 1969.

(C) St. Petersburg State University, 2016 
abgetan, eine Entartung einer monastischen Kultur, die sich zu wahrer poetischer Schönheit im Geiste der Antike nicht hatte erheben können ${ }^{2}$. Scholastik, schlechte Latinität, und zumindest für die protestantischen Apologeten auch der Katholizismus waren, scheint es, in den Jahren von 500 bis 1500 untrennbar miteinander verbunden gewesen, bis das Licht der Reformation auch die lateinische Sprache wiederhergestellt hatte. Theologie- und Philosophiehistoriker wie Lambert Daneau, Flacius Illyricus, Hermann Conring, Adam Tribbechow oder Christian August Heumann hatten dieses Bild gefestigt ${ }^{3}$. Leyser dagegen hatte die bibliographische Arbeit eines Caspar Barth beerbt und die Handschriftensammlungen Marquard Gudes eingesehen, seine Geschichte der mittellateinischen Dichtung summiert auf fast 1000 Seiten nicht nur einen überwältigenden Autorenkatalog, sondern liefert vor allem Editionen mittellateinischer Gedichte, die den Vorwurf der Barbarei bewußt entkräften sollten, die Elegien des Heinrich von Settimello, das Hexaemerongedicht des Hildebert von Lavardin, die Figurengedichte Abbos von Saint-Fleury, aber auch die Metra aus dem, Anticlaudianus' des Alain von Lille.

Begleitend zu seiner Literaturgeschichte hatte Leyser eine Apologie der mittelalterlichen Latinität verfaßt; ihre Zeugnisse waren nicht Produkte einer barbarischen Latinität, sie entsprangen, wie Leyser mit Nachdruck unterstreicht, einer Kultur, die das antike Formenrepertoire ebenso beherrscht hatte, wie sie eine ästhetische Alterität bieten konnte, die Akrosticha und Vagantenstrophen ebenso miteinschloß, wie einen philosophischen Anspruch, der den Worthülsen vieler nachgeborener lateinischer Dichter abgehen mußte. $\mathrm{Daß}$ diese Literatur in ihrem Eigenwert nicht gewürdigt worden war, so formuliert es Leyser deutlich, war ein Ergebnis protestantischer Vorurteile und verdankte sich dem Versuch, sich auf Kosten einer scheinbar dunklen Vergangenheit selbst aufzuwerten. Hatte der Protestantismus das Mittelalter nicht bewußt herabgewürdigt, um in seinem Schatten umso heller strahlen zu können ${ }^{5}$ ? Die Kritiken, die Leysers Arbeit gleich im Anschluß zuteil wurden, waren verheerend ${ }^{6}$. Wie konnte es der Helmstedter Professor wagen, so fragt Jacob Burckhardt im Jahre 1721 in seiner, Geschichte der lateinischen Sprache', die so depravierte Poesie des Mittelalters, die von barbarischer Sprache, theologischer Spekulation und opaken Symbolgeflechten durchdrungene Dichtung dieser Epoche aus dem Orkus zu holen, ja sich sogar um die Ehrenrettung ihrer Verfasser zu bemühen ${ }^{7}$ ? Ein halbes Dutzend Rezensionen und gegen Leyser gerichtete Pamphlete sollte sich in der Folgezeit der Meinung dieses Bibliothekars aus Wolfenbüttel anschließen ${ }^{8}$.

Offensichtlich also hatte, wenn man diesen Äußerungen Glauben schenken darf, in den Augen des 17. und 18. Jahrhunderts eine tiefe Kluft die mittelalterliche und die frühneuzeitliche Latinität voneinander getrennt, offensichtlich auch waren Abgrenzung und Ignoranz die Hauptstrategien der neuzeitlichen lateinischen Poeten gegenüber ihren mittellateinischen Vorgängern gewesen. Diese Untersuchung soll zeigen, daß ein vergleichbares Urteil sich nicht zur Gänze halten läßt, und wie in einer Fallstudie exemplarisch do-

${ }^{2}$ Leyser 1721, Praefatio, fol. 2r-3r, und 1-4.

$3 \mathrm{Zu}$ den protestantischen Polemiken gegen das Mittelalter und seine Sprache im Detail Roling 2015, 275-297. Als Beispiele Tribbechow 1655, dort c. 2, 19-32, oder Clauder 1618, passim.

${ }^{4}$ Leyser 1721, dort die entsprechenen Passagen 302f., 391-408, 1021-1062.

${ }^{5}$ Leyser 1719, 63-69.

6 Ausführlich zur Debatte um Leyser Roling 2016, 303-326, ergänzend auch Zimmermann 1978, 650-669.

7 Burckhard 1721, 63-88.

8 Als Beispiele Bertram 1731, 179-228, und Schmidt 1748, passim. 
kumentieren, wie auch die scheinbar so angegriffene poetische Ästhetik des Mittelalters, oder das, was ihre Gegner als solche dingfest gemacht hatten, die Einbindung spekulativer Theologie, das subtile Spiel mit Typologien, ein scheinbarer Hang zu Neologismen, zumindest subkutan ihre Wirkung weiter entfalten konnten. Zu diesem Zweck wurden hier zwei Beispiele ausgewählt, die beide dem Subgenre der Bibeldichtung entstammen. Auf den ersten Blick scheint es, als müßte sich gerade in dieser konfessionell so wichtigen Gattung die Epochengrenze besonders deutlich abzeichnen. In beiden Konfessionen hatte die Bibeldichtung nach 1450, wie jüngst noch einmal Ralf Czapla hat zeigen können, im Vergleich zum Mittelalter sehr unterschiedliche Richtungen eingeschlagen, vor allem in der protestantischen Domäne mußte, wie man erwarten könnte, der Wille, sich auf die lateinische Dichtung des Mittelalters einzulassen, denkbar gering gewesen sein ${ }^{9}$.

Seit dem 15. Jahrhundert hatten zwei Werke, die in Italien entstanden waren, die lateinische Bibeldichtung der Neuzeit als Paradigmen geprägt, die ,Christias' Marco Vidas, und das Gedicht ,De partu virginis' Jacopo Sannazaros. Beide Werke waren Geniestreiche ihrer Gattung, beide Gedichte zeichnen sich dadurch aus, daß sie das epische Instrumentarium, die Apparaturen, die ihnen die poetische Tradition von Vergil über Lucan bis Statius und Claudian, aber auch christliche Dichter wie Avitus zur Verfügung stellten, zur Gänze in der Komposition ihrer Verse ausschöpften und damit vor allem auf die katholische Bibeldichtung, zuvorderst der Jesuiten, massiven Einfluß ausübten ${ }^{10}$. Die Narrative der Offenbarung mußten nicht in einer linearen, wortgetreuen Abfolge repetiert werden, sie konnten sich in Tableaus frei entfalten. Sannazaro läßt, um nur ein Beispiel zu nennen, den Flußgott Proteus im Jordan einen Ausblick auf die weiteren Begebenheiten des Evangeliums geben ${ }^{11}$, ein Jesuit wie Manuel de Oliveira hatte auch im 17. Jahrhundert in seinem großen Mariengedicht, das sich wie Sannazaro ausschließlich der Empfängnis Mariens widmete, keine Bedenken, den Embryo des Erlösers über hunderte von Versen über die Trinität und die Inkarnation schwadronieren zu lassen ${ }^{12}$.

Es waren diese Ornamente, Variationen und Dramatisierungen, die oft dem bloßen Entertainment geschuldet waren, dem Wunsch des Autors, Langeweile zu vermeiden und seine artistischen Fähigkeiten unter Beweis zu stellen, die man in protestantischen Kreisen für Entgleisungen hielt, vor allem, um ihr ein zur Gänze anderes Konzept von Bibeldichtung gegenüberzustellen. Mit gleicher Logik verurteilt wurden die theologische Überfrachtung von Dichtung, das Anlegen typologischer Symbolsysteme und die Integration von Implementen, die sich nicht unmittelbar aus der Heiligen Schrift stützen ließen. Mit Nachdruck wendet sich der calvinistische Verfasser eines ausgreifenden Evangeliengedichtes, der Steinfurter Johannes Klock, um nur ein Beispiel zu nennen, im Jahre 1602 gegen eine zu weitreichend der Antike verpflichteten Episierung der biblischen Materie und gegen Hybridbildungen aus altem Epos und biblischen Stoffen. Schon Marco Vida, so Klock in der Vorrede zu seiner eigenen ,Christias', hatte den Bogen in seinem gleichnamigen Werk erheblich überspannt ${ }^{13}$. Die organisierte protestantische Bibelepik, wie Klock

9 Als Grundlagenwerk zur Bibeldichtung der Frühen Neuzeit Czapla 2013, passim. Eine Übersicht zur Bibelepik des Mittelalters gibt z. B. Wehrli 1969. Eine Zusammenschau der ganzen Gattung liefert auch Springer 2003.

10 Eine moderne zweisprachige Ausgabe der 'Christias' liegt vor als Vida 1978; und jetzt als Vida 2013.

11 Sannazaro 1987, Liber III, V. 331-485, 74-81.

12 Oliveira de Ferreira 1749, dort zum Embryo Liber II, V.27.

${ }^{13}$ Klock 1602, Praefatio, fol. b5r-b7r. 
und viele seiner Kollegen sie sahen, mußte daher zumindest in ihrem Selbstbild und ihrer Selbstinszenierung in einem erheblichen Maße als humorloser und ironiefreier Rückbau der Errungenschaften der katholischen Bibeldichtung der Italiener verstanden werden, der wieder zur sola scriptura zurückkehren wollte; zugleich war eine Rückkehr zum Mittelalter ausgeschlossen. Die Frontlinie, die hier gezogen wurde, schien sich auf den ersten Blick bruchlos in das zu fügen, was auch die Gegner Leysers gegen dessen Ehrenrettung des Mittelalters geltend gemacht hatten.

\section{Die geheime Erfolgsgeschichte eines Bibelgedichtes aus dem 13. Jahrhundert: Anders Sunesens ,Hexaemeron'}

Ließ sich das harsche Urteil, das in den Augen so vieler Gelehrte der frühen Neuzeit scheinbar eine rigide Schneise zwischen den Epochen und ihren ästhetischen Maximen gezogen hatte, zur Gänze aufrechterhalten? Unser erstes Beispiel schlägt eine Brücke vom 13. ins 17. Jahrhundert, es soll hier nur kurz verhandelt werden, ohne der Komplexität des Werkes dabei gerecht werden zu können. Anfang des 13. Jahrhunderts schreibt der dänische Gelehrte und Bischof von Lund Anders Sunesen ein umfangreiches Bibelgedicht in zwölf Büchern, das ,Hexaemeron', das größte lateinische Gedicht, das in Skandinavien im Mittelalter überhaupt hervorgebracht wurde ${ }^{14}$. Wie sein Freund Saxo Grammaticus war Anders am Hofe des Kriegerbischofs Absalon von Lund beheimatet, studierte wie Saxo in Paris und sollte später die Nachfolge Absalons als Bischof antreten können. Seinen Mäzen beschenkte Anders mit einem mehr als 8000 Verse umfassenden Gedicht, dessen erster Teil aus einer Versifizierung der ersten drei Bücher der Genesis besteht, inklusive einer Fülle von theologischen Exkursen. Die weiteren Werkteile behandeln die Folgen des Sündenfalls, das Wesen der Sünde und die Funktion der Tugenden, die Inkarnation, die Zweinaturenlehre und schließlich die Auferstehung und das Jüngste Gericht. Sunesen spielt mit den klassischen Motiven der mittelalterlichen Bibeldichtung, dem üblichen typologischen Verweissystem, wie es auch andere zeitgenösssische Bibeldichter wie Petrus Riga anzuwenden gewußt hatten, vor allem jedoch integriert der dänische Kleriker die Früchte seiner Pariser Studien. Sten Ebbesen hat nachweisen können, daß vor allem die Quaestionen Stephen Langtons Anders dabei geholfen hatten, die Dreifaltigkeits- und Zweinaturenlehre auf dem Niveau seiner Zeit zu behandeln ${ }^{15}$. Wohl als einziger Bibeldichter macht Sunesen Gebrauch von der aktuellen Suppositionslehre, die über die Porretaner und Abelard in die Theologie Eingang gefunden hatte ${ }^{16}$.

Wichtig ist hier jedoch etwas anderes. Erst im 19. Jahrhundert ist Sunesens Werk zum ersten Mal gedruckt worden, in der Edition von Martin Clarentius Gertz, die 1995 noch einmal mit einer neuen Einleitung versehen wurde ${ }^{17}$. Grundlage der Edition war das einzige mittelalterliche Manuskript, das lange in der Kathedrale von Roskilde aufbewahrt worden war, bis es um 1590 in den Besitz des dänischen Humanisten und Historikers Arild Huitfeldt gelangte ${ }^{18}$. Huitfeldt, eine Schlüsselgestalt der dänischen Geschichts-

${ }^{14}$ Sunesen 1985.

$15 \mathrm{Zu}$ Leben und Werk Sunesens den Überblick von Hørby 1985, Nielsen 1998, passim, und schon Müller 1830, passim. Zu den philosophischen Quellen des ,Hexaemeron' im besonderen Ebbesen 1985.

16 Sunesen 1985, Liber II, V. 782-1227, 99-112.

17 Sunesen 1898.

18 Anders Sunesen, Hexaemeron (Københavens Universitetsbibliotek, MS. E don. Var. 155, $4^{\circ}$ ). Einen 
schreibung, sorgte im gleichen Jahre für die Ausgabe der Landrechte von Schonen, für die sich Sunesen im 13. Jahrhundert ebenfalls verantwortlich gezeigt hatte ${ }^{19}$. Spätestens mit dieser Edition war Sunesen wieder ein bekannter Name in Dänemark geworden, auch wenn es zu einer Druckausgabe des ,Hexaemerons' nicht mehr kam. Ab 1600 begann das Großgedicht des Bischofs im Gelehrtenmilieu Dänemarks und Schwedens dennoch zu kursieren, ungeachtet seiner scheinbaren Fremdheit. Eine grobe Sichtung der frühneuzeitlichen Abschriften zeigt, daß Sunesens Text in dieser Zeit einer ganzen Generation von dänischen Antiquaren und Literaturhistorikern geläufig war. Huitfeldt selbst hatte, wie er am Anfang seiner Edition der ,Landrechte' proklamiert, eine Ausgabe des ,Hexaemeron' geplant ${ }^{20}$, Ole Worm und Stephan Stephanius, zwei der wichtigsten Altertumskundler ihrer Zeit, hatten den Codex ebenfalls autopsiert und einer Edition für wert befunden ${ }^{21}$. Mindestens vier Abschriften werden in diesen Jahren angefertigt, eine von Anders Velleius, einem der weiteren großen Humanisten Dänemarks ${ }^{22}$. In einem Begleitschreiben, das einer der kursierenden Handschriften beigegeben war, konstatiert Otto Sperling, Reichsantiquar Dänemarks, im Jahre 1698, was von dem Gedicht Sunesens zu halten war. Seine Sprache mochte zwar barbarisch sein, wie es dem Zeitalter gebührte, einem Zeitalter, in dem allenfalls, so Sperling, der Phönix Dänemarks, Saxo Grammaticus hervorzuleuchten vermochte. Eine weitere Beschäftigung hatte dem Werk, wie Sperling alludiert, gleichwohl zuteil zu werden; es war zu wichtig, um es ignorieren zu können ${ }^{23}$.

Völlig unbekannt geblieben ist, daß wenige Jahre später auch schwedische Literaturhistoriker und Poetologen begannen, ihrer Begeisterung über Sunesens ,Hexaemeron' Ausdruck zu verleihen. Jacob Wallenius, der als Schwede im schwedischen Greifswald eine Professur für Dichtung innehatte, preist Sunesen Mitte des 18. Jahrhunderts als die große literarische Figur ihrer Zeit, ja als die Initialzündung der lateinischen schwedischen Literatur, dessen vielschichtige und anspruchsvolle Verse gelesen werden sollten. Die schwedische Dichtung mochte mit den Skalden ihren Anfang genommen haben, so Wallenius; in ihrer Literaturgeschichte als ganzer mußte Sunesen als lateinischer Poet eine Schlüsselrolle spielen. Sunesen hatte das bedeutendste, anspruchsvollste und spekulativste Gedicht Schwedens geschrieben, so unterstreicht es Wallenius, den Anfangspunkt seiner lateinischen Literatur, und damit zugleich der Geschichte der schwedischen Lehr- und Bibeldichtung das große Vorbild geliefert ${ }^{24}$. Offensichtlich also konnte sich ein zutiefst der Geisteswelt des frühen 13. Jahrhunderts verpflichtetes lateinisches Großgedicht, den Kämpfen, die ein Polycarp Leyser auszutragen hatte, zum Trotz, großer Wertschätzung erfreuen und wurde gelesen, ja wurde trotz seines theologischen Unterbaus als sprachliches Kunstwerk gewürdigt und kam als Inspirationsquelle in Frage.

glänzenden Überblick über die Manuskript- und Editionsgeschichte des 'Hexaemeron', der wir hier für Dänemark weitgehend folgen, geben Ebbesen und Mortensen in ihrer Einleitung, Sunesen 1985, 19-29. Eine ausführliche Beschreibung der Roskilde-Handschrift liefert auch noch einmal Birger Munk-Olsen 1985.

19 Sunesen 1590.

20 Sunesen 1590, Praefatio, fol. 1r.

21 Worm 1751, Bd. 1, Nr. 264 (Stephanius), 2566.

22 Anders Sunesen, Hexaemeron. Opus metricum de creatione mundu, item lapsu hominis (Københavens Universitetsbibliotek, MS. NKS 121c, $4^{\circ}$ ), fertiggestellt laut Randnotiz am 10. Februar 1590.

${ }^{23}$ Otto Sperling, Epistula ad Olaum Baggem (3. Mai 1698) (ohne Seitenzählung), beigefügt in: Anders Sunesen, Hexaemeron. Opus metricum de creatione mundu, item lapsu hominis (Københavens Universitetsbibliotek, MS. NKS 121c, $4^{\circ}$ ), fol. 2r.

${ }^{24}$ Wallenius, Psilander, Kalén, Sundström, Svensson 1807, Pars II, 34-37. 
Auch Schweden hatte seine frühneuzeitlichen Bibeldichter. 1687 erscheint die ,Bibliska Werld' des Samuel Columbus, der wie viele seiner Zeitgenossen in schwedischer Sprache Anleihen bei Avitus von Vienne gemacht hatte ${ }^{25}$. Zwei Jahre vorher schon war das umfangreichste schwedischsprachige Bibelgedicht in Druck gegangen, ,Guds Werck och Hwila' des Erzbischofs von Uppsala, Haquin Spegel, eines wahren Universalgelehrten. Wie Sunesen liefert Spegel ein Hexaemeron-Gedicht, das entlang der sieben Schöpfungstage im Detail und auf mehr als 12.000 Versen eine theologische Synopse der Schöpfungsgeschichte beschreiben möchte ${ }^{26}$. Spegel dichtet, wie viele physiko-theologische Poeten seiner Zeit, vor dem Hintergrund der Philosophie und Naturwissenschaft seiner Epoche, setzt sich vor allem mit dem Atomismus Gassendis und den Präadamiten LaPeyrères auseinander ${ }^{27}$, doch bemüht sich ihnen einen breiten Konsens aus alten und neuen Bibelkommentaren gegenüberzustellen. Welchem Vorbild Spegel bei allen Unterschieden folgt, verrät er selbst in seiner Einleitung. Philon und Pico waren Stichwortgeber der philosophischen Kommentierung der Offenbarung gewesen, als Dichter jedoch, der sich der Materie auf vergleichbare Weise angenommen hatte, war es Anders Sunesen, der Erzbischof von Lund, in dessen Gefolgschaft sich Spegel sehen wollte ${ }^{28}$. Ein scheinbar zutiefst scholastischer Dichter des 13. Jahrhunderts war einem Dichter des ausgehenden 17. Jahrhunderts also zum entscheidenden Anknüpfungspunkt geworden.

\section{Ein unentdecktes Plagiat im 16. Jahrhundert: Henry Dethicks ,Feriae sacrae' und das ,Hypognosticon' des Laurence von Durham}

Vielleicht war der Kasus des, Hexaemerons' und seines subkutanen Erfolgs den besonderen Vorbedingungen der skandinavischen Marginale verpflichtet, vielleicht auch einfach dem Wunsch nach nationalen Identifikationssymbolen, die sich aus Mangel an eigener antiker Geschichte auch mit mittelalterlichen Paradigmen zufriedengab. Vielleicht aber sprach hier auch eine ästhetische Erfahrung, die stärker war als der humanistische Vorbehalt. Das zweite Beispiel aus der Bibeldichtung soll hier im Vordergrund stehen; es wird deutlich machen, wie weit die subkutane Präsenz des Mittelalters gehen konnte.

\section{Henry Dethick als lateinischer Dichter des 16. Jahrhunderts}

Das frühneuzeitliche elisabethanische England war, wie vor kurzem noch das ,Oxford Handbook of Neolatin' wunderbar hat zeigen können, eine der produktivsten Epochen der neulateinischen Dichtung ${ }^{29}$; dies galt nicht zuletzt, wie J.W. Binns in seiner großen und wertvollen Synopse dokumentiert hat, für die Bibeldichtung ${ }^{30}$. Vielleicht war die religiöse Aufbruchsstimmung dieser Epoche der Gattung besonders förderlich, vielleicht auch sorgten die vielen Erneuerungsbewegungen der Zeit, von den Puritanern bis zu den Quäkern und Diggers für ein besonderes Interesse an der Heiligen Schrift und mußten ihrer Poetisierung nachhaltiger entgegenarbeiten, als in den anderen Ländern Europas. Vor allem die Jahre 1560 bis 1630 sahen eine Fülle von Bibelgedichten. Thomas Drant

\footnotetext{
25 Columbus 1687, fol. Ar-Bv.

26 Spegel 1745 (zuerst 1685).

27 Spegel 1745, 6f.

28 Spegel 1745, Praefatio, fol. ${ }^{\star *}$ r.

29 Haan 2015, 427-444.

30 Binns 1990, 81-83.
} 
sorgte für eine Versadaptation des Ecclesiastes, die er der Regentin zugedachte ${ }^{31}$, William Vaughan für Psalmen- und Canticumparaphrasen ${ }^{32}$. Walter Haddon, ein Jurist der Königin Elisabeth, versifizierte das Buch Hiob, Jesus Sirach und die Apostelgeschichte ${ }^{33}$. Hinzu kamen die Prophetengedichte des Alexander Julius ${ }^{34}$, aber auch eine komplette Versbibel aus der Feder von John Bridges, die nicht mehr als den Text selbst bietet ${ }^{35}$, oder das Bibelcento von Alexander Ross ${ }^{36}$, Werke, denen mit Milton im Zentrum unzählige Bibelepen in englischer Sprache, man denke nur an die ,Davideis' von Abraham Crowley, zur Seite standen. Lateinische Großgedichte wie die ,Poemata sacra' Andrew Ramsays, eine auf 2000 Verse komprimierte Heilsgeschichte, konnten sehr gelungene Passagen enthalten - zu denken ist hier vor allem an ein Epithalamium auf Adam und Eva im catullschen Stil - und haben inzwischen auch in der Forschung Beachtung finden können ${ }^{37}$. Glauben wir dem hervorragenden Referenzwerk James W. Binns, so war es einem weiteren Briten aus dem Umfeld des elisabethanischen Hofes, Henry Dethick, gelungen, das wohl raffinierteste und theologisch anspruchvollste Bibelgedicht seiner Zeit vorzulegen, die ,Feriae sacrae. J. W. Binns beschreibt es als „outstanding exampel“ seiner Gattung und preist Dethick, „a man, who organized his material more profoundly than most“. Im ganzen, so Binns, hatte Dethick ein Meisterwerk des 16. Jahrhunderts vorgelegt, „a serious and devout work, which sets more store by its doctrine than by its appeal as poetry". Es war, so Binns, "preeminent amongst the numerous works of biblical latin, produced to exemplify the theories of the supremacy of divine poetry, held by Dethick himself. ${ }^{38}$ "

Wer war nun dieser großartige Poet und welche Werke hatte er noch verantwortet? Über sein Leben informiert uns der Universalbiograph seiner Zeit, Anthony a Wood ${ }^{39}$, weiteres Material hatte Binns selbst zusammentragen können ${ }^{40}$. Dethick hatte in Oxford studiert, wo er $1572 \mathrm{im}$ Alter von 25 Jahren graduierte. Er war aus hofnaher Familie, sein Vater Gilbert Dethick und sein Onkel William waren,Garter King of Arms' der Krone gewesen. Dethick selbst bescherten sein Studium und, so darf vermutet werden, seine Reputation als Dichter, den Posten eines Erzdiakons in Carlisle, wo er 1613 verstarb. Um 1572 hatte Dethick, wie Binns, der Neuherausgeber des Textes zeigen konnte, eine ,Oratio in laudem poeseos' in Druck gebracht. Dichtung, so proklamiert der junge Student der Theologie, hatte den Menschen zu zivilisieren; sie hatte sich auch der Allegorien und poetischen Bilder zu bedienen, um den Lesern zur Moralität zu verhelfen ${ }^{41}$.

Die folgenden Jahre sehen Dethick als Lieferanten diverser Widmungsgedichte, also einen Poeten, der sich wie zu erwarten um Kontakt zu Kollegen bemühte und begann ein Netzwerk aus Mitstreitern aufzubauen. Er schenkt der lateinischen Übersetzung des ,Cortigiano', die Bartholomew Clerke vorlegt hatte, einige Distichen, die Clerke als neuen
${ }^{31}$ Drant 1572.
32 Vaughan 1597.
${ }^{33}$ Haddon 1576.
34 Julius 1611.
35 Bridges 1604.
36 Ross 1638. Zu diesem Werk z. B. Döpp 2000, dort bes. 3-7.
37 Thanner 2005, dort zur Catull-Rezeption 366-373.
38 Binns 1990, 104-108.
39 Wood 1820, Bd. 1, 208.
40 Binns 1990, 146-148.
${ }^{41}$ Dethick 1999, 26-49, dort bes. 42-45. Zur Entstehungsgeschichte der 'Oratio' auch schon Binns 1975. 
Cicero feiern ${ }^{42}$. Auch die ,Dialektik' von Richard Stanyhurst, die ebenfalls in dieser Zeit erschienen war, erhält eine Versdedikation, die eine genaue Lektüre des Werkes anmahnen möchte ${ }^{43}$. Dethick erscheint in beiden Werken innerhalb einer Galerie von ehrgeizigen jungen Männern, die sich alle in der Entourage von Großaristokraten der Zeit bewegten, Edward de Vere, dem 17. Earl von Oxford und William Cecil Lord Burghley. Beide wiederum hatten schon zum Bekanntenkreis seines Vaters gehört. Eine Neuausgabe des ,Gardeners Labyrinth' von Thomas Hill, eines Handbuchs der Gartenkunde, scheint das Ouevre des jungen Dethick im Jahre 1575 abzurunden ${ }^{44}$. Vor uns sehen wir also einen Dichter, der sich, wie so viele seiner Epoche um Geltung bei Hofe bemühte und das damit einhergehende symbolische Kapital zu kumulieren hatte. Erwartet wurde nun, so scheint es, das Großgedicht, das die in der ,Oratio' fünf Jahre zuvor proklamierten Ansprüche einlösen konnte; Dethick liefert es mit seinen ,Feriae sacrae ${ }^{45}$. Gewidmet waren die acht Bücher Bibeldichtung dem schon genannten Lord Burghley, dem Schatzmeister der Königin, der sich zu diesem Zeitpunkt auf der Höhe seines Ruhms befand. Die Jahre zuvor schon war der Lord als leidenschaftlicher Verteidiger der anglikanischen Sache in Erscheinung getreten, einer Verteidigung, die, wie allgemein bekannt, mit der Hinrichtung der Maria Stuart ihren Höhepunkt erreichen sollte. Burghley war zweifelsohne ein gebildeter, vielleicht sogar des Griechischen kundiger Mann; ob er die zahllosen Bücher, die ihm zugedacht wurden, gelesen hatte, sei dahingestellt.

Dethicks Widmung an Burghley, aber auch die sich ihr anschließenden Dedikationsgedichte seiner Kollegen in Oxford zeigen, daß Dethick mit seiner Arbeit an einem Unterstützernetzwerk erfolgreich gewesen war. Widmung und Gedichte gehen gleichermaßen auf den Wert der ,Feriae sacrae' ein und feiern die 4000 Verse in elegischen Distichen als gehaltvollen Ausdruck der geistlich-moralischen Dichtung, der sich Dethick scheinbar verschrieben hatte, und die sich elegant von der weltlichen Poesie abzugrenzen wußte. Dethick selbst sagt von sich, er habe die Bibel denkbar gründlich gelesen, und wie jemand, der in der Sonne gebräunt wurde, selbst die Farbe der Offenbarung angenommen. Die amplitudo des royalen Schatzmeisters, sie werde verherrlicht, nicht durch die credula phantasmata der paganen Götterwelt, sondern die credita charismata der Heiligen Schrift. Keine fabulae enthalte sein Gedicht, sondern die historiae, keine carmina ethnici, sondern carmina Christiani, die dem Verwalter des weltlichen Schatzes Ihrer Majestät einen geistigen Schatz bescheren konnten ${ }^{46}$. Im gleichen topischen Gefüge, das an das bekannte Proöm des Juvencus zu seiner Evangeliendichtung erinnert, bewegen sich auch die Dedikationsgedichte, die den Mehrwert der geistlichen Dichtung hervorheben. Laurence Humphrey, der damalige Präsident des Magdalene Collegue in Oxford, feiert die ,Feriae sacrae' mit horazischen Sentenzen, weil sie nicht die profanen nugae lieferten, sondern heilige Mußestunden ${ }^{47}$, Christopher Jonson, damals Headmaster des Winchester Collegue, lobt Dethick in catullschen Elfsilblern, weil er nicht die Amoris faces oder die Venus furtiva, die Irrfahren des Odysseus oder die comoedia prisca besungen hatte, sondern die

${ }^{42}$ Clerke 1577, fol. Pp3v. Die erste Auflage hatte die Verse noch nicht enthalten.

43 Stanyhurst 1570, fol. bv.

${ }^{44}$ Hill 1577. Gewidmet war auch dieses Werk, wie die Praefatio zeigt, William Cecil Lord Burghley.

45 Dethick 1577.

46 Dethick 1577, fol. A2rf.

47 Dethick 1577, fol. A3r. 
Schätze der Offenbarung, die den Geist der göttlichen Liebe atmeten ${ }^{48}$. Ein Peter Fry kann als Widmungsbeiträger noch hinzufügen, daß Dethicks Verse nicht mit dem Schlangengift der stupra nefanda aufwarteten, sondern den Mysterien der Heiligen Schrift ${ }^{49}$, ein John Williams schließlich, daß hier nicht der vergilianische Iopas crinitus das Chaos und die Elemente schilderte, sondern der Dichter die christliche Schöpfung aus dem Nichts $\mathrm{zu}$ beschreiben wußte ${ }^{50}$. Auch die übrigen zwei Widmungsgedichte können diese Ideen nur variieren. Alle Paratexte bezeugen also, wie sehr hier ein geistliches Werk vorgelegt wurde, das in seiner Abkehr von allem Weltlichen Neues und zugleich zutiefst Altes und Ehrwürdiges in die Welt trug, die Wahrheit der Heiligen Schrift.

\section{Vierhundert Jahre aktuell: Ein erfolgreiches Plagiat}

Gerne nun würde man in den topischen Lobgesang der Dedikationsgedichte miteinstimmen und auch der spärlichen Sekundärliteratur beipflichten, wenn Dethicks Verse nicht ein großes Manko hätten. Sie waren ein vollständiges Plagiat. In seinem Inhalt, seiner sprachlichen Substanz, ja im Fall von mehr 80 Prozent des Versbestandes Wort für Wort, rekrutierten sich die ,Feriae sacrae' Dethicks aus dem ,Hypognosticon' des Laurence von Durham, einem Bibelgedicht, das jener um 1130 in England geschrieben hatte. Vereinfacht gesagt, Dethicks elegische Distichen waren das ,Hypognosticon', ein in seiner Epoche außerordentlich erfolgreiches Bibelgedicht, dessen Verse durchaus Eingang in die Standardpoetiken seiner Zeit gefunden hatten. Anders als so viele spätantike Bibeldichter, man denke an Sedulius oder Avitus, war dieser Text auch nach 1500 nicht gedruckt worden, auch wenn, wie sich überprüfen läßt, das Vorhandensein der Handschriften in den englischen Bibliotheken von mehreren Katalogen nach dem Tod Dethicks bezeugt wird. Der Katalog der Cotton Library Thomas Smiths verzeichnet das ,Hypognosticon' ebenso ${ }^{51}$, wie Edward Bernards großer ,Catalogus der englischen und irischen Bibliotheken', der die Handschriften in den Oxford Colleges ebenso auflistet wie die Handschrift von Durham, die auch den beiden modernen Editionen als Grundlage gedient hatte ${ }^{52}$. Schon in der unmittelbaren Zeit Dethicks war Laurence mit seinem Gedicht den einschlägigen englischen Bibliographen wie John Bale oder Johannes Pitseus geläufig gewesen ${ }^{53}$. Heute liegt das ,Hypognosticon' in zwei Ausgaben vor, einer von Maria Liguori Mistretta aus dem Jahre 1941 und einer zweiten von Susanne Daub aus dem Jahre 200254. Quellenstudien und eine Einordnung des Gedichtes in die Geschichte der mittelalterlichen Bibeldichtung sind bisher erst in sondierender Form geleistet worden ${ }^{55}$. Irgendwann um 1570 mußte Dethick auf das Großgedicht des Laurence von Durham gestoßen sein und sah seine Chance gekommen; vermutlich hatte er, soweit

${ }^{48}$ Dethick 1577, fol. A3rf.

49 Dethick 1577, fol. A4v.

50 Dethick 1577, fol. A4v-A5v.

51 Smith 1696, 36, 114.

52 Bernard 1697, 4, Nr. 42, 247, Nr. 937.

53 Bale 1557, Centuria II, 196; und Pitseus 1619, Bd. 1, 221.

${ }^{54}$ Laurentius von Durham 2002, und Laurence von Durham 1941. Schwester Liguoris Editionsleistung wird in der Ausgabe Daubs leider nicht gewürdigt. Laurentius war ein vergleichsweise produktiver Dichter gewesen, unter seinen weiteren Werken, die ebenfalls nicht den Weg in den Frühdruck gefunden hatten, z. B. Laurentius von Durham 1880, und Laurence von Durham 1969.

${ }_{55}$ Als wesentliche, leider überwiegend an klassischen Similien interessierte Studie bisher Daub 2005, und mit diesem Werk identisch Daub 2003. Eine Studie zu Laurence im Vergleich mit Petrus Riga und Alexander von Ashby liefert Dinkova-Bruun 2008. 
es sich an den Varianten derzeit erahnen läßt, eine der Oxforder Handschriften gewählt und für den Druck vorbereitet.

Plagiate waren im 16. Jahrhundert keine Seltenheit. Bei meiner Arbeit an den JonaDichtungen der Frühen Neuzeit bin ich auf einen Text aus dem Jahre 1557 gestoßen $^{56}$, den man schon zwei Jahre nach seiner Abfassung erneut vorlegen konnte ${ }^{57}$, und sogar auf einen zweiten in alkäischen Strophen, der nach 1590 sogar zweimal plagiiert und zu allem Unglück sogar völlig deckungsgleich demselben Adressaten dargeboten wurde ${ }^{58}$. Dethick war in seinem geistigen Diebstahl professioneller vorgegangen. Als geübter Dichter hatte er durchaus Verse optimiert und Junkturen des Laurence von Durham, wie er glaubte, zum Besseren verändert. Sein Gedicht, das sich um 450 Jahre versetzt als modernes Bibelgedicht deklariert, gibt uns die singuläre Gelegenheit, zu fragen, wieviel Mittelalter im ausgehenden 16. Jahrhundert, exemplarisch in der Bibeldichtung, präsentiert werden konnte, ohne damit eine Kollision der Ästhetiken zu provozieren. Wer beide Texte, das Original und Dethicks Plagiat, miteinander vergleicht, stellt mit Verblüffung fest, wie wenig Dethick zum Ende als für seine eigene Zeit und seine Adressaten als unangemessen eingestuft hatte. Kollektiv streichen mußte Dethick natürlich alle expliziten Selbstapostrophierungen des Autors, die Laurence selbst beim Namen genannt hatten und das Proöm. Interessant für die Frage nach der Akzeptanz und der ästhetischen Diskursfähigkeit des Mittelalterlichen über die Jahrhunderte hinweg sind jedoch die weiteren Eingriffe, die Dethick vornimmt. Welche Passagen des ,Hypognosticon' erschienen ihm nach 400 Jahren untragbar und konnten, wie er glaubt, einem mit der Reformation vertrauten Umkreis nicht mehr zugemutet werden?

Das ,Hypognosticon' hatte auf neun Bücher verteilt eine typologie-getränkte Synopse der Heilsgeschichte gegeben, so wie andere Bibeldichter der Zeit, Heinrich von Augsburg $^{59}$, Fulco von Beauvais ${ }^{60}$, oder der bekannteste, Petrus Riga, dessen ,Aurora' die größte Streubreite besa ${ }^{61}$. Laurence hatte die Allegorien der einschlägigen Bibelkommentare integriert und sich auch theologischen Exkursen nicht verweigert. Das ganze Werk folgt der Gliederung in die Epochen ,Vor', ,Während des' und ,Nach dem Gesetz' und damit einem augustinischen Raster ${ }^{62}$. Buch 1 hatte Schöpfung und Sündenfall und die Zeit nach der Sintflut verhandelt, Buch 2 die Zeit bis zum Turmbau von Babel, doch zugleich einen langen Exkurs zur Theodizee miteingeschlossen, Buch 3 gebührte den Patriarchen, Abraham, Isaak, Jacob, Joseph und seinen Brüdern und verhandelte dann den Auszug der Israeliten aus Ägypten und ihre Wanderung durch die Wüste. Vor allem der letzte hatte Laurence zu allerlei allegorisierenden Betrachtungen Anlaß geboten, die das seit Avitus etablierte Bildmaterial, inklusive der Pentateuch-Exegesen Bedas und Rhabans zur Geltung brachte. Tatsächlich streicht Dethick aus diesen ersten drei Büchern nicht mehr als 50 Verse. Die kurz angerissene Elementenlehre, die bei Laurence im Zentrum der Schöpfungserzählung stand, die augustinische Schilderung der Vertreibung aus dem Paradies, aber auch die Analyse der Erbsünde bedurften nach 400 Jahren keiner Verän-

56 Roling (im Druck).

57 Hermann 1555, plagiiert von Hübner 1557.

58 Saxo 1590, plagiiert von Poletanus 1594, und noch einmal von Mundorph 1599.

59 Heinrich von Augsburg 1956, Text 161-226.

${ }^{60}$ Fulco von Beauvais 1960, dort zu Leben und Werk Fulcos, der Laurence sicher geläufig war, die Einleitung 40-46.

${ }_{61}$ Petrus Riga 1965.

62 Zum augustinischen Schema des 'Hypognosticon' auch Daub 2005, 15-31. 
derung ${ }^{63}$. Hundert Verse hatte Laurence mit Anselm von Canterbury darauf verwandt, zu erklären, wie göttliche Vorherbestimmung, Freiheit, zeitliche begrenzte Sünde und ewige Verdammnis sich in Einklang bringen ließen; Dethick läßt sie unverändert, denn auch die Theologie der eigenen Zeit, wie ein kurzer Blick in reformierten Dogmatiken, genannt sei Girolamo Zanchi, die in England kursierten, zeigen kann, hatte sich noch immer dieser Autoritäten bedient ${ }^{64}$. Auch den zweiten langen Exkurs, zum Verhältnis von pietas und iustitia im göttlichen Heilsplan, beläßt Dethick zur Gänze. Kein Theologe seiner Zeit hätte ihn beanstanden können ${ }^{65}$. Gleiches galt in den Augen Dethicks scheinbar auch für alle Typologien. Was aber läßt Dethick weg? Knapp dreißig Verse hatte Laurence, als er die späte Schwangerschaft der Sara erklärt, der Spermaqualität alter Männer geschenkt und ihrer physiologischen Begründung ${ }^{66}$. Vielleicht wollte sie Dethick dem Schatzmeister der ewig jungfräulichen Königin als Lektüre einfach nicht zumuten.

In Buch 4-6, die dem Gesetz zugedacht waren, hatte Laurence nach einer theologischen Exposition die Gebote der Torah traktiert und nach ihrer typologischen Bedeutung gefragt, die Epoche der Richter bis zu Saul geschildert, die Könige Israels, David Konflikte mit seinen Söhnen, die weiteren Regenten, die Vertreibung der Israeliten, die Propheten, Daniel und die Bücher Esra, und schließlich sogar die Makkabäer. Auch hier ist der Befund bei Dethick bemerkenswert. Der englische Dichter hält es in Buch 4 weder für notwendig, die langen Ausführungen des ,Hypognosticon' zur Korruption der menschlichen Natur einer ernsthaften Kürzung zu unterziehen ${ }^{67}$, noch das breite Geflecht an Allegorien, das Laurence entfaltet, als er den grünenden Stab Aarons oder die eherne Schlange des Mose erklärt ${ }^{68}$. Im darauffolgenden Buch bleiben auch die langen Verspassagen zur Sünde König Davids und zum Tode Abschaloms unangetastet ${ }^{69}$, vor allem aber die stark an Minne-Diskurse der Zeit angelegten Monologe, die Laurence der Gestalt Amons in den Mund gelegt hatte, jenem Sohn Davids, der sich in seine Schwester Tamar verliebt hatte. Ihn läßt Laurence in langen Verspassagen räsonnieren, ob man der Liebe nachgeben sollte oder nicht; die gerechte Strafe für seinen Liebesfuror ereilt den Prinzen unverzüglich ${ }^{70}$. Auch ein langes Referat aus dem dritten Buch Esra, das die Gewalt von Königen, Frauen, Wein und Wahrheit gegeneinander ausspielt, geht dem Fluß des Textes nicht verloren ${ }^{71}$. Weniger deutlich als die protestantische Kirche hatte die anglikanische Kirche dieser Zeit die deuterokanonischen und ganz apokryphen Texte aus dem Kanon ausgeschieden. Wieder erweist sich für den frühneuzeitlichen Bibeldichter nicht die Allegorese oder die Moralisation als anstößig, sondern die explizite Sprache. Dethick tilgt Laurences bildmächtige Schilderung der sogenannten Schandtat von Gibea,

${ }^{63}$ Dethick 1577, Liber I, fol. 5r-7r, dazu Laurentius von Durham 2002, Liber I, V. 1-164, 73-78.

${ }^{64}$ Dethick 1577, Liber II, fol. 12r-13v, dazu Laurentius von Durham 2002, Liber II, V.53-96, 84-86, V. 127-228, 87-91.

${ }^{65}$ Dethick 1577, Liber II, fol. 15vf., dazu Laurentius von Durham 2002, Liber II, V.322-366, 94-96.

${ }^{66}$ Laurence von Durham 2002, Liber III, V.309-336, $107 f$.

${ }^{67}$ Dethick 1577, Liber IV, fol. 30rf., dazu Laurentius von Durham 2002, Liber IV, V. 1-68, 124-126.

68 Dethick 1577, Liber IV, fol. 32vf., dazu Laurentius von Durham 2002, Liber IV, V.219-234, 131f.

${ }^{69}$ Dethick 1577, Liber V, fol. 42r-43v, dazu Laurentius von Durham 2002, Liber V, V.267-380, $158-162$.

${ }^{70}$ Dethick 1577, Liber V, fol. 47r-48v, dazu Laurentius von Durham 2002, Liber V, V.567-720, 169-173.

${ }^{71}$ Dethick 1577, Liber VI, fol. 51v-54r, dazu Laurentius von Durham 2002, Liber VI, V.71-268, $182-190$. 
der Vergewaltigung und Ermordung einer Levitenfrau aus Richter $19^{72}$, und die homoerotisch konnotierte Beschreibung der Liebe zwischen David und Jonathan ${ }^{73}$. Letztere mochte zwar in der Zisterziensertheologie eines Aelred von Rievaulx keine Fragen aufwerfen, die Theologie des 16. Jahrhunderts hätte ihr jedoch vielleicht mehr Zweideutigkeit angedeihen lassen, als Dethick es beabsichtigen konnte.

Auf den ersten Blick erscheint es, als könnte fast die gesamte poetisierte Theologie des Hochmittelalters, mitsamt ihrem allegorischen Apparat, in Dethicks U-Boot in die Neuzeit geschafft werden. Die letzten drei Bücher des ,Hypognosticon' läßt Laurence mit einer neuen, fast 400 Verse langen Exposition beginnen, die noch einmal die Notwendigkeit der Inkarnation im Heilsgeschehen unterstreicht, dann folgt die Geburt Christi und ein Zusammenschnitt aus den Evangelien bis zur Himmelfahrt. Den Abschluß des Werkes formte die Investitur der Sakramente, die Apostelgeschichte und die Frühzeit der Kirche, von den ersten Märtyrern bis zu den Heiligen der englischen Kirche, allen voran St. Cuthbert, dessen Leben Beda ja schon versifiziert hatte. Erst jetzt, als sich das Werk dem Ende neigt, beginnt Dethick massiv in den Text einzugreifen. Noch vergleichsweise ungeschoren kommen Laurences Referate aus der Theologie des Anselm von Canterbury davon und seine Satisfaktionslehre, die das fleischgewordene Wort erklären konnten ${ }^{74}$, dann aber hatte es Laurence gewagt, fast 150 Verse der Bedeutung der Heiligen Jungfrau Maria zu widmen ${ }^{75}$. Ihre alttestamentlichen Präfigurationen in der Bundeslade und dem brennenden Busch, ihre pietas und potentia, ihr gesamter Tugendkatalog, die Laurence den Werken des Anselmschülers Eadmer von Canterbury entnommen hatte, aber auch die wunderbare Weise, in der sich, so Laurence, in ihrem Uterus factor und factura vereinigen konnten, eingeschlossen die iustificatio, die Rechtfertigung, die Christus mit ihr der Welt zuteilwerden ließ; all diese Insignien des Katholizismus waren 17 Jahre nach dem Tod Maria der Katholischen in einem England, das die Papstkirche hinter sich gelassen hatte, nicht vermittelbar ${ }^{76}$. Dethick konnte sie Burghley keinesfalls vorlegen. Damit war mehr als das halbe siebte Buch der Vorlage weggefallen.

Auch das achte Buch säuberte Henry Dethick von allen mariologischen Versatzstücken, um dann den Faden mit der gewöhnlichen Heilsgeschichte wiederaufzunehmen ${ }^{77}$. Auffällig ist auch hier eine Reihe von signifikanten Auslassungen, die zeigen, daß Dethick sein Plagiat wohlüberlegt in Angriff genommen hatte. Gestrichen werden in der Passionsgeschichte 30 Verse zur Kreuzverehrung, die bei Laurence an Rhabanus Maurus erinnert hatten ${ }^{78}$. Als der Heiland zu den Patriarchen hinabsteigt, muß auch die Typologie einige Federn lassen. Jonas als Typus des Erlösers war, wie zu erwarten, noch vermittelbar, Rahab

${ }^{72}$ Laurentius von Durham 2002, Liber IV, V. 443-60, 140.

73 Laurentius von Durham 2002, Liber V, V. 53-82, 151.

${ }^{74}$ Dethick 1577, Liber VII, fol. 58r-60v, dazu Laurentius von Durham 2002, Liber VII, V.1-120, 201-205.

75 Laurentius von Durham 2002, Liber VII, V. 121-266, 205-210.

76 Als Beispiel einer Auslassung Laurentius von Durham 2002, Liber VII, V.241-248, 209f.: Sic de carne caro, de virgine virgo creatur, / Virgoque mater ei materiesque fuit. / Nam de virginea sacra carne caruncula sumpta / Effigiatur abhinc in nova membra deo. / Corpus id optatum post augmentatur in ortum, / Expletisque novem mensibus exit homo. / Carnis nube latens sol exiit ille perhennis, / Sponsus ut e thalamo, virginis ex utero.

77 Dethick 1577, Liber VIII, fol. 62rf., dazu weit ausführlicher Laurentius von Durham 2002, Liber VIII, V. 1-68, 215-217.

78 Dethick 1577, Liber VIII, fol. 63r-64r, dazu Laurentius von Durham 2002, Liber VIII, V.69-234, 217-223, die Verse zur veneratio crucis V. 194-224, 222 f. 
als Typus der Kirche war es offensichtlich nicht ${ }^{79}$. Auch einige Passagen zur Rechtfertigung in der Liebe Gottes schienen der iustificatio im Glauben, wie sie die zeitgenössischen Protestanten proklamiert hatten, zuwiderzulaufen ${ }^{80}$; ebenso die Beteiligung der Engelchöre während der Himmelfahrt Christi ${ }^{81}$.

Das letzte Buch, das Dethick mit dem achten verschmelzen muß, um die Einheit der Buchlängen einigermaßen zu wahren, verliert ebenfalls fast die Hälfte seines Umfangs. Auch hier hatte Laurence viele Typologien zur Geltung gebracht, die Dethick nicht beanstanden mußte. Einige der allfälligen Präfigurationen des Altarsakramentes konnten erhalten bleiben, andere, der Vergleich des Wassers, das Mose aus dem Felsen geschlagen hatte, mit dem Blut des Erlösers, fallen der Zensur des selbsternannten Bibeldichters zum $\mathrm{Opfer}^{82}$. Nicht die Allegorie und die Vielheit der Schriftsinne, sondern die Sakramente in ihrer Siebenheit waren im Jahre 1575 zum eigentlichen Problem geworden. Noch deutlicher zeigt sich dies, wie vielleicht zu erwarten, bei der bis ins Mittelalter reichenden weiteren Heilsgeschichte. Dethick kann den Aposteln noch den entsprechenden Raum zubilligen, fast das einzige Mal im ganzen Werk fühlt er sich sogar berufen, noch ein eigenes Distichon zum Heiligen Stephanus beizusteuern ${ }^{83}$. Die Kette der englischen Heiligen, St. Alban oder Oswald, auch die Kirchenlehrer bis zu Anselm von Canterbury, die Laurence noch hatte folgen lassen, werden gestrichen ${ }^{84}$. Ans Ende seiner ,Feriae' stellt Dethick einen Abschnitt aus dem ,Hypognosticon', den Laurence eigentlich als Prolog des neunten Buches vorgesehen hatte ${ }^{85}$, eine lange Warnung vor dem falschen otium, mit dem das wahre, das auf Gott ausgerichtete otium kontrastieren mußte, eben jene gottesfürchtige Variante der feriae, die dem Werk Dethicks den Titel gegeben hatte ${ }^{86}$. Zumindest er selbst, so läßt es der Dichter sein Publikum wissen, hatte seine Zeit sinnvoll angewendet.

79 Dethick 1577, Liber VIII, fol. 64v, dazu weit ausführlicher Laurentius von Durham 2002, Liber VIII, V. 235-372, 222-224, V.299-320, 224f. Bei Dethick heißt es als Maximum an Typologie lediglich: Lector in his el quaere favis, in pane petatur / Mica, trahantur abhinc interiora nucis: / Nux sit Adam, sit panis Adam, sit Adam favus idem, / Christus enim nucleus, mica, mel eius adest. / Corpus, plaga, Deus, fuit archa, fenestra, columba, / Gratia certa manens, extat oliva virens. / Est Isaac dietas, aries caro, crimina vepres, / Spiritus ignis, ovis cornua, ligna crucis. / Navis item Ionae, crux Christi, mors, mare piscis, / est tumulus, tumulus sentit is, ille feram.

${ }^{80}$ Dethick 1577, Liber VIII, fol. 64vf., dazu weit ausführlicher Laurentius von Durham 2002, Liber VIII, V.373-398, 224f.

81 Dethick 1577, Liber VIII, fol. 65rf., dazu weit ausführlicher Laurentius von Durham 2002, Liber VIII, V.321-352, 226f.

82 Dethick 1577, Liber VIII, fol. 66r, dazu weit ausführlicher Laurentius von Durham 2002, Liber IX, V 189-334, 234-240.

83 Dethick 1577, Liber VIII, fol. 66vf., dazu Laurentius von Durham 2002, Liber VIII, V.361-366, 241. $\mathrm{Zu}$ Stephanus heißt es bei Dethick: Quae sit vera fides qua possit quisquis placere / Altissimo, forti disserit ore Stephan. / Obturant aures, lapidoso grandine pressus / proque suis moriens hostibus ille rogat.

${ }^{84}$ Laurentius von Durham 2002, Liber IX, V.367-516, 241-246.

${ }^{85}$ Laurentius von Durham 2002, Liber IX, V. 1-56, 228-230.

${ }^{86}$ Dethick 1577, Liber VIII, fol. 68rf. Hier heißt es, aus Laurence genommen, für Dethick am Ende titelgebend: Accipiter tibi saepe liber, sit Biblia tantum / Pro praeda canibus, carmina saepe iuvant. / Pro Sathana, Christum, reprobo, consortia iusti / Quaere, nec his cessas tu feriare modis. / Sed iam propositum retinet stylus, atque libellus / Huic operi metas, ultimus iste dabit. 


\section{Fazit}

Um einiges deutlicher als die autoritative Referenz Anders Sunesens im zutiefst protestantischen Schweden zeigt Henry Dethick, wie wenig zum Ende die vermeintlich neue Ästhetik, die auf Reduktion im Sinne des sola scriptura-Prinzips oder eine Episierung im antiken Stil bedacht war, die Kopräsenz scheinbar zeitenferner und in ihrem Wesen mittelalterlicher Typologien ausschließen mußte. Nur einige dogmatische Gehalte verlangten nach einer Transformierung, Maria, Heiligenverehrung oder die Sakramente, das Textgebäude des ,Hypognosticon' mit einen erheblichen Teil seiner Allegorien konnte die Reise durch die Epochen jedoch fast zur Gänze unbeschadet überstehen; es war trotz aller Veränderungen in der Erwartungshaltung des Publikums nicht veraltet. Es wundert vielleicht nicht, daß Polycarp Leyser, dem Verfasser der mittellateinischen Literaturgeschichte, beide Werke, Sunesen wie Laurence von Durham, geläufig waren. Sunesen kannte Leyser aus der dänischen Fachliteratur, Laurence durch die Lektüre der englischen Manuskriptkataloge und der einschlägigen Bibliographien ${ }^{87}$. Auch wenn Leyser keine Auszüge aus diesen Manuskripten offeriert, hätten beide Autoren sicher seinen Geschmack gefunden. Sunesen und vor allem Laurence von Durham dokumentieren jedoch auch, daß die Mauer, die scheinbar die neue Epoche der lateinischen Literatur ab 1500 von der mittelalterlichen trennte, durchlässiger war, als ihre Apologeten in ihrem späthumanistischen Selbstverständnis proklamiert hatten. Das scheinbar Überwundene, und Veraltete konnte seinen Ort bewahren, sein Provokationspotential, war aller Korrekturbedürftigkeit zum Trotz, geringer, als man hätte glauben müssen.

Diese Beobachtungen, die hier zunächst nur für das begrenzte Terrain der Bibeldichtung gemacht werden, doch die sich möglicherweise leicht auch auf andere Gattungen wie die Lehrdichtung ausdehnen lassen, sollten sich mit zwei Forschungsdesideraten verbinden, die dabei offen zutage treten. Zum ersten: Die Kopräsenz von gedruckten und handschriftlichen Materialien auch ab 1600 bedarf noch immer weiterer Untersuchungen. Auch in der Gelehrtenwelt dieser Jahrhunderte konnten kursierende und kopierte Handschriften noch immer eine gewaltige Wirkung entfalten. Und zum zweiten: Die Auseinandersetzung mit lateinischer Literatur sollte sich ihre Gegenstände nicht von künstlichen Epochengrenzen diktieren lassen, denn sie verdanken sich, wie gezeigt, in ihren Anfangsgründen oft Motiven, die wir heute nicht mehr nachvollziehen können.

\section{Literaturhinweise}

Bale, J. Scriptorum illustrium maioris Brytanniae, quam nunc Angliam et Scotiam vocant, Catalogus. Basel 1557.

Bernard, E. Catalogi librorum manuscriptorum Angliae et Hiberniae in unum collecti. Oxford 1697

Bertram, J.F. Dissertatio Anti-Lyseriana prior de vera medii aevi barbarie, adversus V.C. Polycarpi Lyseri Dissertationem de ficta medii aevi barbarie, novis accessionibus praesertim thesisbus de Theologia scholastica aucta, in: ders., Meletemata literaria varii haud iniucundi nec inutilis argumenti. Braunschweig 1731.

Binns, J. W. The first appearance in print of an Elizabethean Treatise. The Library, 1975, 30, 199-210.

Binns, J.W. Intellectual Culture in Elizabethan and Jacobean England. The Latin Writings of the Age. Leeds 1990.

Bridges, J. Sacro-sanctum Novum Testamentum in hexametros versus translatum. London 1604.

${ }^{87}$ Leyser 1721, 429-431, 1000. 
Burckhard, J. De linguae latinae quibus in Germania per XVII saecula amplius usa ea est fatis commentarii. Wolfenbüttel 1721.

Clauder, J. Oratio saecularis de inculta et horrida superioris pontificiae excultaque et florida nostrae Lutheranae aetatis latinitate. Altenburg 1618.

Clerke, B. Balthasaris Castilionis comitis de curiali sive aulico libri quatuor. London 1577.

Columbus, S. Bibliska Werld, in: Bibliska Werld, sampt andre hans poetiske Skriffer, med Flijt samblade och andre resan uglagde. Stockholm 1687.

Czapla, R. G. Das Bibelepos in der Frühen Neuzeit. Zur deutschen Geschichte einer europäischen Gattung. Berlin 2013.

Daub, S. Vergil und die Bibel als verschränkte Prätexte. Ein poetisches Experiment. Rheinisches Museum 2003, 146, 85-102.

Daub, S. Von der Bibel zum Epos. Politische Strategien des Laurentius am geistlichen Hof von Durham. Köln 2005.

Dethick, H. Feriae sacrae octo libris comprehensae. London 1577.

Dethick, H. Oratio in laudem poeseos, in: James W. Binns (Hg.), Latin treatises on poetry from Renaissance England. Signal Mountain 1999, 26-49.

Dinkova-Bruun, G. Rewriting Scripture. Latin Biblical Versification in the Later Middle Ages. Viator 2008, 39, 263-284.

Döpp, S. Virgilius Evangelisans. Zu Praefatio und Prooemium von Alexander Ross' Christias (1638). (Nachrichten der Akademie der Wissenschaften in Göttingen. I. Philologisch-historische Klasse 6 [2000]). Göttingen 2000.

Drant, Th. In Salomonis regis et praeconis Ecclesiasten, seu de vanitate mundi concionem sapientissimam et celeberrimam paraphrasis poetica. London 1572.

Ebbesen, S. Hexaemerons svære passager: kvæstioner og teologisk logik, in: Sten Ebbesen (Hg.), Anders Sunesen: stormand, teolog, administrator, digter. Kopenhagen 1985, 137-150.

Fulco von Beauvais, Utriusque de nuptiis Christi et Ecclesiae libri septem, hg. von Sister Mary Isaac Jogues Rousseau, Washington 1960.

Haan, E. The British Isles, in: S. Knight, S. Tilg (Hgg.), The Oxford Handbook of Neo-Latin. Oxford 2015, 427-444.

Haddon, W. Poematum libri duo, hg. von Th. Hatcher. London 1576.

Heinrich von Augsburg. Planctus Evae, in M. L. Colker (Hg.): Traditio 1956, 12, 149-230.

Hermann, J. Historia Ionae prophetae versibus elegiacis reddita. Wittenberg 1555.

Hill, Th. The Gardeners Labyrinth, containing a discourse of the gardeners life, hg. von Henry Dethick, London 1577.

Hübner, P. Historia Ionae prophetae versibus elegiacis reddita. Wittenberg 1557.

Hørby, K. Anders Sunesens liv, in: Sten Ebbesen (Hg.), Anders Sunesen: stormand, teolog, administrator, digter. Kopenhagen 1985, 11-25.

Julius, A. Paraphrasis prophetiae Chabakkuki poetica, Edinburgh 1610; und ders., Paraphrasis prophetiae Maleaci poetica. Edinburgh 1611.

Klock, J. Christiados priscae et novae libri XII. Oberursel 1602.

Laurentius von Durham, Dialogi, hg. von James Raine, Durham 1880.

Laurence von Durham, The Hypognosticon of Lawrence of Durham, a preliminary text with an introduction by Maria Mistretta Liguori, Washington 1941.

Laurence von Durham, Consolatio de morte amici, hg. von Ulrich Kindermann, Diss. Erlangen — Nürnberg 1969.

Laurentius von Durham, Hypognosticon. Gottes Heilsplan — verdichtet, hg von Susanne Daub, Erlangen Jena 2002.

Leyser, P. Dissertatio de ficta medii aevi barbarie imprimis circa poesin latinam speciminibus non inelegantibus carminum editorum et ineditorum corroborata, Historiae poetarum medii aevi praemissa. Helmstedt 1719.

Leyser, P. Historia poetarum et poematum medii aevi decem post annum a nato Christo CCCC seculorum. Halle 1721.

Müller, P.E. Vita Andreas Sunonis Archiepiscopi Lundensis Programma. Kopenhagen 1830.

Mundorph, Ch. Paraphrasis lyrica Prophetiae Jonae Amithae. Wittenberg 1599.

Munk-Olsen, B. Roskildehåndschrift af Hexaemeron, in: Sten Ebbesen (Hg.), Anders Sunesen: stormand, teolog, administrator, digter. Kopenhagen 1985, 233-242. 
Nielsen, T. K. Anders Sunesen. Danmark og verden i 1200-tallet. Odense 1998.

Oliveira de Ferreira, M. de, Poema epicum de conceptione Beatae Mariae. Coimbra 1749.

Petrus Riga Aurora. Biblia versificata. A Verse Commentary on the Bible (2 Bde.), hg. von Paul E. Beichner. Notre Dame 1965.

Pitseus, J. Relationes historicae de rebus anglicis quatuor partes complectens (2 Bde.). Paris 1619.

Poletanus, G. Paraphrasis lyrica Prophetiae Jonae Amithaei. Wittenberg 1594.

Roling, B. Saeculum barbaricum - Frühneuzeitliche Stereotypen in der Philosophiegeschichtsschreibung des Mittelalters. Frühmittelalterliche Studien 2015, 49, 275-297.

Roling, B. Die Geburtswehen der mittellateinischen Philologie: Polycarp Leyser IV. und das Mittelalter, in: G. Frank, V. Leppin (Hgg.), Die Reformation und ihr Mittelalter. Stuttgart 2016, 303-326.

Roling, B. Held wider Willen: Jonasepik zwischen Mittelalter und Konfessionalisierung, in: B. Quast, S. Spreckelmeier (Hgg.), Inkulturation - Literarische Strategien bibelepischen Schreibens in Mittelalter und Früher Neuzeit (im Druck).

Ross, A. Virgilii evangelisantis Christiados libri XIII. London 1638.

Sannazaro, J. De partu Virginis, hg. von Ch. Fantazzi, A. Perosa. Florenz 1987.

Saxo, D. Paraphrasis lyrica Prophetiae Ionae Amithaei. Wittenberg 1590.

Schmidt, J.J. Barbariem medii aevi non fictam, sed veram contra virum clarissimum Polycarpum Lyserum. Nordhausen 1748.

Smith, Th. Catalogus librorum manuscriptorum bibliothecae Cottoniae. Oxford 1696.

Spegel, H. Guds Werck och Hwila: the är Hela Werldenes underwärda Skapelse uthi sex dagar af then alsmächtiga guden fulbordad. Nörrköping 1745.

Springer, C.P.E. The Biblical Epic in Late Antiquity and the Early Modern Period: The Poetics of Tradition, in: Zweder von Martels - Victor M.Schmidt (Hgg.), Antiquity renewed. Late Classical and Early Modern Themes. Leuven, Paris 2003, 103-126.

Stanyhurst, R. Harmonia seu catena dialectica. London 1570.

Sunesen, A. Leges provinciales terrae Scaniae ante annos 400 latinae redditae, hg. von Arild Huitfeldt. Kopenhagen 1590.

Sunesen, A. Hexaemeron libri duodecim, hg. von M. C. Gertz. Kopenhagen 1898.

Sunesen, A. Hexaemeron (2 Bde.), hg. von M.C.Gertz, S.Ebbesen, L. B. Mortensen (Corpus Danicorum philosophorum medii aevi 3, 4). Kopenhagen 1985.

Thanner, T. Vom Apfelbaum im Paradies zum Streit der Töchter Gottes: Andrew Ramsay, Poemata sacra (Edinburgh 1633). Einleitung, Text, Übersetzung, Kommentar und Interpretation. Frankfurt 2005.

Tribbechow, A. De doctoribus scholasticis et corrupta per eos divinarum humanarumque rerum scientia liber singularis. Giessen 1655.

Vaughan, W. Erotopaignion pium. London 1597.

Vida, M. G. The Christiad. A Latin-English Edition, hg. von G. C. Drake, C. A. Forbes. Carbondale, London 1978.

Vida, M.H. Christias. Einleitung, Edition, Übersetzung, Kommentar, hg. von E.von Contzen, R.F.Glei, W. Polleichtner (2 Bde.). Trier 2013.

Wallenius, J. Psilander, C. U. (resp.), Kalén, M. (resp.), Sundström, P. (resp.), Svensson, N. (resp.). Schediasma de Poetis Suecanis celebrioribus. Greifswald 1807.

Wehrli, M. Sacra Poesis: Bibelepik als europäische Tradition, in: ders., Formen mittelalterlicher Erzählung. Aufsätze. Zürich 1969, 51-71.

Wood, A. Athenae Oxonienses. An exact history of all the writers and bishops who have had their education in the University of Oxford (4 Bde.). London 1820.

Worm, O. Olai Wormii et ad eum doctorum virorum Epistolae (2 Bde.). Kopenhagen 1751.

Zimmermann, H. 'De medii aevi barbarie. - Ein alter Gelehrtenstreit, in: K. Hauck, H. Mordek (Hgg.), Geschichtsschreibung und geistiges Leben im Mittelalter. Festschrift für Heinz Löwe zum 65. Geburtstag. Köln 1978, 650-669.

For citation: Roling B. Von Anders Sunesen zu Henry Dethick: Kontinuitäten des Mittellateinischen in der Frühen Neuzeit. Philologia Classica 2016, 11(1), 131-147. DOI: 10.21638/11701/spbu20.2016.112 


\section{ОТ АНДЕРСА СУНЕСЕНА ДО ГЕНРИ ДЕТИКА: НЕПРЕРЫВНОСТЬ СРЕДНЕВЕКОВОЙ ЛАТЫНИ В РАННЕЕ НОВОЕ ВРЕМЯ}

\section{Бернд Ролинг}

В начале 18 в. Поликарп Лейзер, ученый из Хельмштедта, опубликовал свою «Историю средневековой поэзии», приложив к ней защиту средневековой латыни от обвинений в «варварстве». Труд молодого ученого получил негативную оценку его современников: средневековая латинская поэзия, по мнению его оппонентов, перегружена неологизмами, типологией и схоластической спекуляцией. В статье приводятся, тем не менее, два примера из столь неоднозначно оцениваемого жанра, как библейская поэзия, показывающие, что средневековая латынь, хотя и была презираема гуманистами, без серьезных препятствий воспринималась поэтами XVII и XVIII веков. Первый - поэма о творении «Гексаэмерон» Андерса Сунесена, епископа Лунда в Дании (1201-1224); подвергшись осуждению в качестве «варварской», поэма, тем не менее, не только стяжала похвалы как шедевр, но и была авторитетной в шведской библейской поэзии 18 в. Еще более разителен пример «Feriae sacrae» англо-латинского поэта Генри Детика (1577), образец спекулятивной библейской поэзии, представляющий собой полный плагиат «Гипогностикона», поэмы Лоренса из Дарема (XII в.). Сравнивая оригинальную версию с ее «адаптацией» эпохи раннего Нового времени, можно показать, что основная часть текста была использована в XVII веке в качестве «современной» и отвечавшей амбициозным стремлениям плагиатора. Лишь отдельные разделы поэмы были им исключены, как более не соответствующие церковным догмам эпохи Елизаветы.

Ключевые слова: Средневековая латинская поэзия, Андерс Сунесен, Генри Детик, латинская литература Нового Времени. 